Ann. Biol. anim. Bioch. Biophys., I968, 8 (4), 579-580.

\title{
" EFFET MATERNEL " ASSOCIÉ A L'ETAT HOMOZYGOTE POUR LE GÈNE W (PATTES ET PEAU BLANCHES) CHEZ LA POULE
}

\author{
P. MÉrat \\ Station centrale de Génétique animale, \\ Centre national de Recherches zootechniques, 78 -Jouy-en-Josas \\ Institut national de la Recherche agronomique
}

Sur II générations d'une population expérimentale (souche Jouy) où les allèles $\mathrm{W}$ (peau blanche) et $w$ (peau jaune) étaient maintenus en ségrégation, la descendance des accouplements où la mère était homozygote pour le gène $\mathrm{W}$ $\left(\sigma^{\top} \mathrm{W} w \times q \mathrm{WW}\right.$ et $\delta$ ww $\left.\times \phi \mathrm{WW}\right)$ avait un poids moyen à 8 semaines inférieur à celui des croisements réciproques $(\hat{\sigma} \mathrm{WW} \times q \mathrm{~W} w$ et $\hat{\sigma} \mathrm{WW} \times$ 우 rw respectivement).

Les moyennes sur l'ensemble des années et par type de croisement sont, à titre indicatif, les suivantes (tabl. I) :

\section{TABLEAU I}

Poids à huit semaines en relation avec le type de croisement pour le gène $W$ (total de II années, souche Jouy)

\begin{tabular}{|c|c|c|c|c|}
\hline \multirow[b]{2}{*}{ Type de croisement } & \multicolumn{2}{|c|}{$\sigma^{t}$} & \multicolumn{2}{|c|}{ 우 } \\
\hline & $\begin{array}{l}\text { Nombre de } \\
\text { descendants }\end{array}$ & $\begin{array}{l}\text { Poids moyen } \\
\text { (g) }\end{array}$ & $\begin{array}{l}\text { Nombre de } \\
\text { descendants }\end{array}$ & $\begin{array}{l}\text { Poids moyen } \\
\text { (g) }\end{array}$ \\
\hline 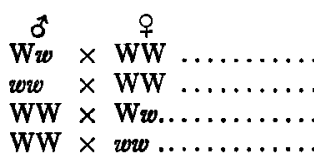 & $\begin{array}{l}630 \\
667 \\
442 \\
449\end{array}$ & $\begin{array}{l}827,0 \\
834,0 \\
875,8 \\
885,5\end{array}$ & $\begin{array}{l}627 \\
621 \\
496 \\
496\end{array}$ & $\begin{array}{l}705,4 \\
687,0 \\
734,1 \\
739,0\end{array}$ \\
\hline
\end{tabular}

La différence moyenne intra-année, sur l'ensemble des données, entre croisements de mère WW et croisements réciproques, est voisine de $50 \mathrm{~g}$ chez les coquelets et $35 \mathrm{~g}$ chez les poulettes.

Un écart de même sens se retrouve pour les femelles (seules comparées) issues de 
mères WW ou de mères d'un autre génotype, dans quatre autres populations élevées au domaine du Magneraud : deux souches de pondeuses (2 générations analysées pour chacune) et deux souches " chair " (4 et 2 générations respectivement). Ici, la comparaison n'a pu être faite sur des croisements réciproques. Le génotype des enfants au locus W diffère donc en même temps que celui des parents, mais, par ailleurs, une comparaison directe (MÉRAT, données non publiées) ne permet pas de déceler de différences de performances associées au génotype des zygotes.

Les poids moyens à 8 semaines de ces souches allaient de 550-60o $\mathrm{g}$ à I rooI $200 \mathrm{~g}$. La différence moyenne intra-année, à l'avantage de la descendance des mères non homozygotes $W W$, était comprise entre ${ }_{5} \mathrm{~g}$ et $60 \mathrm{~g}$ approximativement, suivant la population.

Dans chaque souche, une analyse de variance a été faite sur les moyennes de familles de même père, avec les facteurs contrôlés " type de croisement relatif au locus W " et " année ". Au total, les différences liées au génotype des parents pour $\mathrm{W} / w$ sont hautement significatives ; il en est de même de plusieurs de ces populations considérées séparément $(\mathrm{P}<0,00 \mathrm{I}$ pour l'une des souches « chair »).

En outre, la ponte d'automne et d'hiver des poulettes de mère WW, des mêmes cheptels, était plus faible que celle des filles de mère Ww ou wre, sans exception. En particulier, la différence est de même sens dans les II générations de la souche Jouy $(P<0,00 I)$. L es écarts vont de 3 à Io $p$. Ioo environ en pourcentage journalier de ponte, suivant la population. La maturité sexuelle, relevée dans la souche Jouy, est retardée d'une semaine à peu près lorsque la mère est WW.

Le poids des poules adultes de mère WW est, également, inférieur (de 40 à $200 \mathrm{~g}$ approximativement). Dans deux des souches, dont la population Jouy, la différence est significative au seuil i p. roo.

Ces résultats ont une incidence pratique pour les sélectionneurs produisant des poulets de chair à peau blanche : le plus souvent, une souche mâle Cornish à peau jaune est croisée à une souche femelle dans laquelle le gène $W$ a été introduit par un croisement. Il paraîtrait préférable, d'après les données présentes, de fixer plutôt l'allèle $\mathrm{W}$ dans la souche " père ).

Reçu pour publication en juillet 1968.

\section{SUMMARY}

“ MATERNAL EFFECT" ASSOCIATED WITH HOMOZYGOSITY FOR GENE W (WHITE LEGS AND SKIN) IN THE HEN

In Ir generations of a population in which the $\mathrm{W}$ (white skin) and $w$ (yellow skin) alleles are kept segregating, the progeny of dams homozygous for $W$ had a significantly lower performance for 8 week weight than the reciprocal crosses. The same holds for laying performance of publets.

Similar differences were found in 4 other strains.

It appears from our results that for breeders usually producing crosses of a male yellowskinned strain with a female strain where $W$ was introduced by a cross, the fixation of $W$ in the male strain would be more advisable. 